\title{
Optical tests as the basis for formulating mathematical models of the opening delay of CIDI injectors
}

The main objective of this research was an attempt to evaluate the delay times of the actual needle opening of the diesel injectors in relation to the time of triggering the current control signals opening the solenoid and piezoelectric high-pressure injectors of diesel engines. The conducted tests take into account the variability of fuel injection pressure and backpressure prevailing in the operational chamber of the engine. To determine accurately the time of actual injection start, the optical tests analysing the image of the injector tip were used. Such high resolution images were obtained by high-speed recording with a frequency of $250 \mathrm{kHz}(\Delta t=0.004 \mathrm{~ms})$. Based on a comparison of the results obtained, it was found that the maximum delay time of fuel injection for a piezoelectric diesel injector is about $12 \%$ shorter than for a solenoid injector.

Experimentally obtained results of the injection time delay were used as a basis to formulate mathematical models describing the delay of the real fuel injection in relation to the signal controlling the opening of the diesel injectors. These models take into account the dependence of the injector reaction from the injection pressure and the backpressure in the operational chamber of the engine. The correctness of the obtained models is confirmed by acceptable values of the determination coefficient (for solenoid injector - 0.6, for piezoelectric injector - above 0.8 - for correlation of injection delay and backpressure).

Key words: diesel fuel, direct injection, injection delay, optical research, solenoid and piezoelectric injector

\section{Introduction}

High pressure liquid fuel injection used in common rail system requires precise dosing of fuel through the injectors. Currently solenoid or piezoelectric injectors are used both in spark-ignition and diesel engines. Petrol injection systems operate at lower values of injected fuel pressure than $\mathrm{CI}$ engine systems, but fuel injection times are similar. This tendency causes that the requirements of engine injectors with SI and CI are similar. They should demonstrate short response time to input signals (controlling signals) and, at the same time, short closing times of injectors with high repeatability of the voltage-current signals.

The studies on the two types of injectors were conducted by $\mathrm{Yu}$ et al. [7], where the base fuels were kerosene and diesel. Using only electrical signals triggering injector (control signal) and changes in the flow of fuel from the injector, the fuel flow delay was specified. Permanent delay values regardless of the fuel injection pressure (were obtained 60 or $100 \mathrm{MPa}$ ). For piezoinjectors this time amounted to $0.45 \mathrm{~ms}$, while for electromagnetic injectors to $0.55 \mathrm{~ms}$. In addition, a significantly higher fuel flow rate at lower pressures of injected fuel were observed. It was stated that such an increase in the rate of the fuel flow brings tangible benefits in actual engines in the form of better atomization and evaporation of the fuel. Optical studies of the authors concerned only observation of the development of fuel spray planes and not delay assessment of fuel flow from the injector.

Research carried out by Zhou et al. [8] concerned the possibility of determining fuel flow rate for each of the little openings in the injector. The studies used a conventional injection system with a serial pump used in CI engines of the off-road vehicles. Based on the results of the fuel flow, the diversity of the fuel flow time for individual openings can be determined. Those differences amount to
2 degrees of rotation of the pump shaft at the speed range for this pump of 800-1000 rpm.

In research conducted by Salvador et al. [3] the opening delay was specified as the difference of the time of injector triggering (start of energizing) and the flow of fuel from the injector was determined in the system for measuring fuel flow rate (start of injection). The tests were conducted for fuel pressures ranging from 30 to $180 \mathrm{MPa}$ and at different fuel temperatures. It was shown that with increasing fuel pressure the injection delay decreases. It amounts to 0.24$0.28 \mathrm{~ms}$. This delay increases with decreasing fuel temperature. At fuel temperature of $253 \mathrm{~K}$ and $\mathrm{P}_{\text {inj }}=40 \mathrm{MPa}$ it is about $0.35 \mathrm{~ms}$.

Optical studies on piezoelectric injectors were conducted by Magno and et al. [2]. These studies focused on the analysis of the difference in the rate of fuel flow from a new injector and an injector contaminated by long-lasting operation. Injection rate measurements have proven to be a useful tool to demonstrate that a contaminated injector operates with a delay in relation to the new injector. It was also found that both injectors inject a similar amount of fuel, but with altered characteristics of the injection. In fact, contaminated injector injects a bigger amount of fuel as a pilot dose, but smaller as the main dose. The studies didn't analyze, however, the delay of the fuel flow from the injectors.

Duan et al. [1] while conducting test on the new type of electromagnetic injectors obtained a hydraulic delay of $0.406 \mathrm{~ms}\left(\right.$ at $\mathrm{P}_{\text {inj }}=250 \mathrm{MPa}$, and energizing time $=1.5 \mathrm{~ms}$ ). Compared to currently produced injectors, the hydraulic delay values were reduced by $11.5 \%$ (Cummins injector) and $24.8 \%$ in relation to the Bosch injector (for which the studies with the same values of configurable parameters were conducted).

Analysis of the literature showed a large number of publications concerning the determination of: 
1) electrical signals parameters,

2) hydraulic parameters of piezo- and solenoid injectors; however, there is a lack of works indicating the assessment of the actual beginning of the flow from the injector while taking into account the electric and hydraulic delay in relation to the emergence of electrical signals controlling the beginning of the injection.

Previous works of the authors of this article [4-6] concerned the initial research to this problem. Optical tests conducted within these studies on the delay of hydraulic flow of fuel were carried out at the filming speed of $50 \mathrm{kfps}$ (thousands of images per second). They confirmed the applicability of optical testing to determine the time of the start of fuel flow from the injector; it is recommended, however, to use a higher time resolution. More accurate research with an increased rate of filming for injectors in CI engines were partly completed in the current studies, in which the speed of filming has been greatly increased up to $250 \mathrm{kfps}$.

\section{The research problem}

The aim of this publication is the elemental analysis of the impact of injection parameters on the fuel injection delay (fuel pressure and air backpressure). On the basis of this analysis the mathematical description of the fuel injection delay relating to high-pressure electromagnetic and piezoelectric diesel injectors was developed. The acquisition of such a model is crucial in terms of issues concerning controlling combustion engine with the CIDI.

\section{Research methodology}

It was decided that the above formulated task would be resolved with the use of experiment involving optical methods. A constant volume chamber (CVC) was used to simulate the backpressure in the cylinder during fuel injection, as well as the combination of the system for measuring fast-varying process and the high-speed camera for fast registration of photos. Such a combination of apparatus for the acquisition allowed conducting synchronized measurements of the electrical signals at the injector and the observation of the physical appearance of drops in the measuring chamber. So prepared test bench is shown in Fig. 1. Parameters of the tested injectors are shown in Table 1, and the technical specifications of the recording apparatus are shown in Tables 2 and 3. For the purpose of these tests the frequency of $250 \mathrm{kHz}(\Delta \mathrm{t}=4 \mu \mathrm{s})$ was used for recording optical tests and of $500 \mathrm{kHz}(\Delta \mathrm{t}=2 \mu \mathrm{s})$ for recording electrical signals. To connect and synchronize both data types a flash light of diode was used.

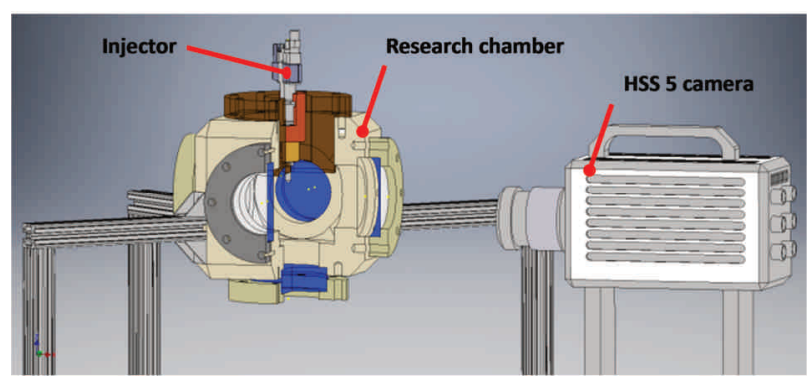

Fig. 1. Diagram of the test bench for optical analysis of the delay of fuel injection
As a control apparatus a sequencer was used generating a TTL signal $(0-5 \mathrm{~V})$ on the basis of which the systems triggering injection, the camera and the diode are controlled. For the tests were used two different injector common rail systems: the first was the piezoelectric injector, the other - solenoid injector (both by Bosch). Parameters of the injectors tested are presented in Table 1.

Table 1. Characteristics of the injectors

\begin{tabular}{|l|c|c|}
\hline Parameter & Solenoid & Piezoelectric \\
\hline Static flow & $520 \mathrm{~cm}^{3} / \mathrm{min}$ & $705 \mathrm{~cm}^{3} / \mathrm{min}$ \\
\hline $\begin{array}{l}\text { Angle of the cone of fuel } \\
\text { spray }\end{array}$ & $162 \mathrm{deg}$ & - \\
\hline Number of orifices & 9 & 8 \\
\hline Fuel injection pressure & $25-180 \mathrm{MPa}$ & up to $200 \mathrm{MPa}$ \\
\hline
\end{tabular}

Table 2. Details of the optical setup and devices employed to evaluate the fuel injection delay

\begin{tabular}{|l|c|}
\hline Camera & LaVision High Speed Star 5 \\
\hline Light source & Halogen lamp $-2 \times 500 \mathrm{~W}$ \\
\hline Camera lens & Nikon Nikkor \\
\hline Lens features & $50 \mathrm{~mm}-\mathrm{f} / 1.4$ \\
\hline Image size (pix) & $128 \times 16$ \\
\hline Frame rate (fps) & 250,000 \\
\hline Test repetitions & 2 \\
\hline
\end{tabular}

Table 3. Details of the fast-varying setup and devices employed to evaluate the fuel injection delay

\begin{tabular}{|l|c|}
\hline System & AVL Indimodule \\
\hline Analog input channels & $8(0-10 \mathrm{~V})$ \\
\hline Maximum sampling rate & $800 \mathrm{kHz}$ \\
\hline Software & $\begin{array}{c}\text { AVL Indicom - recording system } \\
\text { AVL Concerto - data analysis system }\end{array}$ \\
\hline On board memory & $64 \mathrm{MB}$ \\
\hline
\end{tabular}

For such configured test bench test points that corresponded to the operation points of the injector during supplying the combustion engine were selected. The injection pressure, injector opening time (due to the difference of injector parameters, it was decided to use the longer opening times of opening for electromagnetic injector) and backpressure of the medium into which fuel was injected were subjected to analysis. The range of variation of these parameters is shown in Table 4 . Each test point was repeated to confirm the result obtained.

Table 4. Points of measurements

\begin{tabular}{|l|c|}
\hline Parameter & Value \\
\hline Injection pressure $\mathrm{P}_{\text {inj }}$ & $30-120 \mathrm{MPa} ; \Delta \mathrm{P}=30 \mathrm{MPa}$ \\
\hline Back-pressure in the $\mathrm{CVC}_{\mathrm{b}}$ & $2.5-4 \mathrm{MPa} ; \Delta \mathrm{P}=0.5 \mathrm{MPa}$ \\
\hline Energizing time ET & $0.2 ; 0.4 ; 0.6 \mathrm{~ms}$ for piezo-injector \\
& $0.3 ; 0 ; 5 ; 0.7 \mathrm{~ms}$ for solenoid injector \\
\hline No. of iterations & 2 for each point \\
\hline
\end{tabular}

Delay for these tests was defined as a component of two factors. The first was the time of current rise in the system (defined as electrical delay $-t_{e}$, Fig. 2) and the other - the appearance of drops in the chamber, called a hydraulic delay $-t_{h}$. Only the sum of these two delays gives a complete picture of the entire delay between the injection request signal and its physical realization (according to Eq. 1). 


$$
t_{d}=t_{e}+t_{h}
$$

Synchronization of the tests (optical and electrical) made it possible to determine the total time of delay in operation of injectors $\left(t_{d}\right)$ : electrical delay is defined as the time between the appearance of the TTL control signal, and the beginning of the current rise $\left(\mathrm{I}_{\mathrm{inj}}\right)$ in the system (Fig. 2). The hydraulic delay is defined as the time from the moment of the current rise to the appearance of the first drops in the measuring chamber. So defined hydraulic delay time, due to the need of the use of two separate methods of recording (electric signal and photos) is described by Eq. 2 .

$$
\mathrm{t}_{\mathrm{h}}=\mathrm{t}_{1}+\mathrm{t}_{2}
$$

The course of the current-voltage characteristics of the investigated piezoelectric injector of diesel is shown in Fig. 3.

The time from the moment of current rise until the moment the diode flash was defined as $t_{1}$. This interpretation made it possible to combine optical tests and registration of electrical signals.

The method of processing the results of optical tests is illustrated in Fig.4. First, the background image was subtracted in order to remove random reflections. Then the frame with a lit diode was found - from this frame the time in optical tests was measured. The other important recorded frame is the moment of fuel flow from the injector $(100 \mu$ s in Fig. 4). The time between these frames is marked as $t_{2}$. The sequence showing the determination of the time $t_{2}$ is presented in Fig. 4.

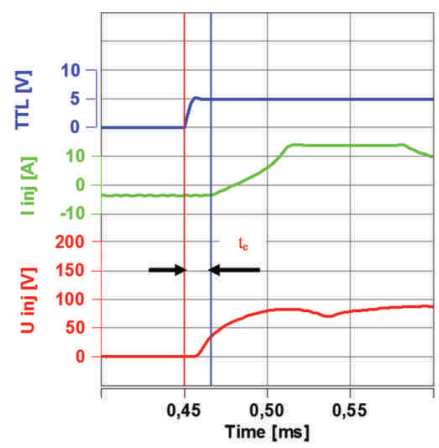

Fig. 2. A method for determining the electric delay for solenoid injector (based on data from solenoid injector: $\mathrm{ET}=0.5 \mathrm{~ms}, \mathrm{P}_{\mathrm{inj}}=120 \mathrm{MPa}$ $\left.\mathrm{P}_{\mathrm{b}}=40 \mathrm{MPa}\right)$

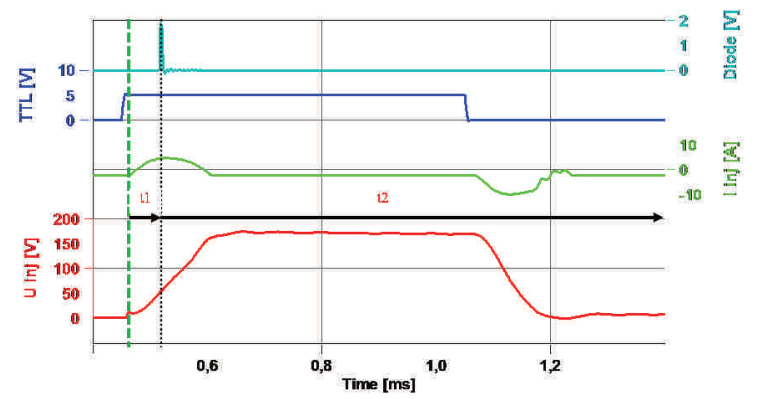

Fig. 3. A method for determining the time $t_{1}$ for the hydraulic delay (based on data from piezoelectric injector: $\mathrm{ET}=0.6 \mathrm{~ms}, \mathrm{P}_{\mathrm{inj}}=30 \mathrm{MPa}, \mathrm{P}_{\mathrm{b}}=25 \mathrm{MPa}$ )

\begin{tabular}{|c|c|c|c|}
\hline Lp. & time/action & Lp. & \multicolumn{2}{|c|}{ time/action } \\
\hline 1 & & 20 & $68 \mu \mathrm{s}$ \\
\hline 2 & & $\downarrow$ & \multicolumn{2}{|c|}{$\downarrow$} \\
\hline 3 & & 27 & $96 \mu \mathrm{s} \quad$ injection \\
\hline 4 & $4 \mu \mathrm{s}$ & 28 & $100 \mu \mathrm{s}$ \\
\hline 5 & $8 \mu \mathrm{s}$ & 29 & $104 \mu \mathrm{s}$ \\
\hline$\downarrow$ & & 30 & $108 \mu \mathrm{s}$ \\
\hline 18 & $60 \mathrm{us} \quad$ diode blink & 31 & $112 \mu \mathrm{s}$ \\
\hline 19 & $64 \mu \mathrm{s}$ & 32 & $116 \mu \mathrm{s}$ \\
\hline
\end{tabular}

Fig. 4. Optical research algorithm to achieve time $t_{2}$ (based on data from solenoid injector: $E T=0.5 \mathrm{~ms}, P_{\text {inj }}=120 \mathrm{MPa}, \mathrm{P}_{\mathrm{b}}=40 \mathrm{MPa}$ )

\section{Investigation of injection delay for piezo- and solenoid injectors}

The tests carried out indicated the difference in the delay of both electromagnetic and piezoelectric injectors. Basing on the analysis of the obtained results, the factors were defined and their influence on the delay of injection. In the analysis the difference of the results at a level of $4 \mu \mathrm{s}$ (resulting from the frequency of recording) should be treated as the same result due to the measurement uncertainty. The effect of such assumptions is demonstration of actual trends arising from the phenomena taking place during injection. A summary of the results obtained is shown in Fig. 5 for the solenoid injector and in Fig. 6 for piezoelectric injector. In the summary, the points with the same energizing time of the injection were located in one chart, allowing analysis the influence of injection pressure and back pressure (Fig. 7, Fig. 8).

Based on the results obtained, the operation of the electromagnetic injector can be assessed showing higher repeatability in relation to the piezoelectric injector. The 
increase of the injection pressure was indicated as the main value determining an injection delay for electromagnetic injector. Comparing the test points it can be concluded that there is a significant randomness in operation of the piezoelectric injector. The analysis failed to show a trend for the impact of the triggering signal duration on the injection delay. It is possible to characterize the results for both injectors at injection pressure of $30 \mathrm{MPa}$ - for this scope in both cases the increasing backpressure has influence on reduction of the delays.

The above-presented methodology of analysis was considered inadequate; therefore, it was decided to complement it with additional assessment tools based on mathematical statistics in order to obtain a more comprehensive assessment of the results obtained.

It was decided to use, subsequently:

- a mean value,

- medium value,

- dominant value.

After complementing the analysis with the indicated statistical options, again the summary of the results was carried out, but in this case adopting different criteria for the presentation of data. Summary to verify the impact of the injection time on the delay is shown in Figs 7 and 8, respectively, for solenoid and piezoelectric injector.
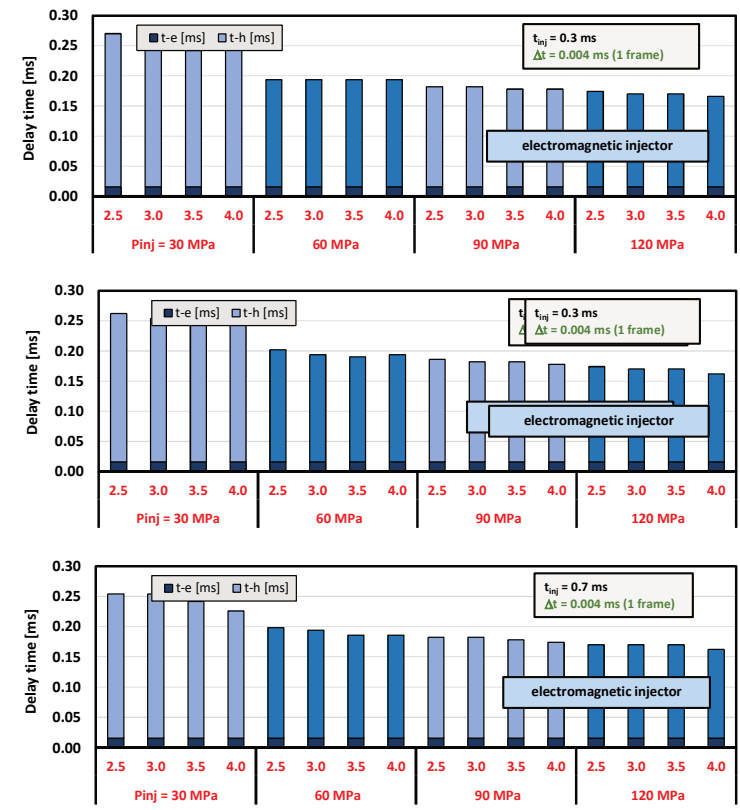

Fig. 5. Analysis of the times of injection delay at variable pressure of fuel injection and backpressure of air during solenoid injector operation

The summary for electromagnetic injector does not show any higher impact of the time of input signal of injection opening on the injection delay, which is a problem while defining the relationships. Overlapping of statistical values marked on the graphs with horizontal lines (Figs 7 and 8) confirms the inability to indicate the specific dependencies. However, a tendency for the lowest and highest injection pressure is visible, indicating that the increase of the backpressure in the chamber causes the acceleration of the injector opening. Two middle pressure injection settings, however, do not confirm this dependency.

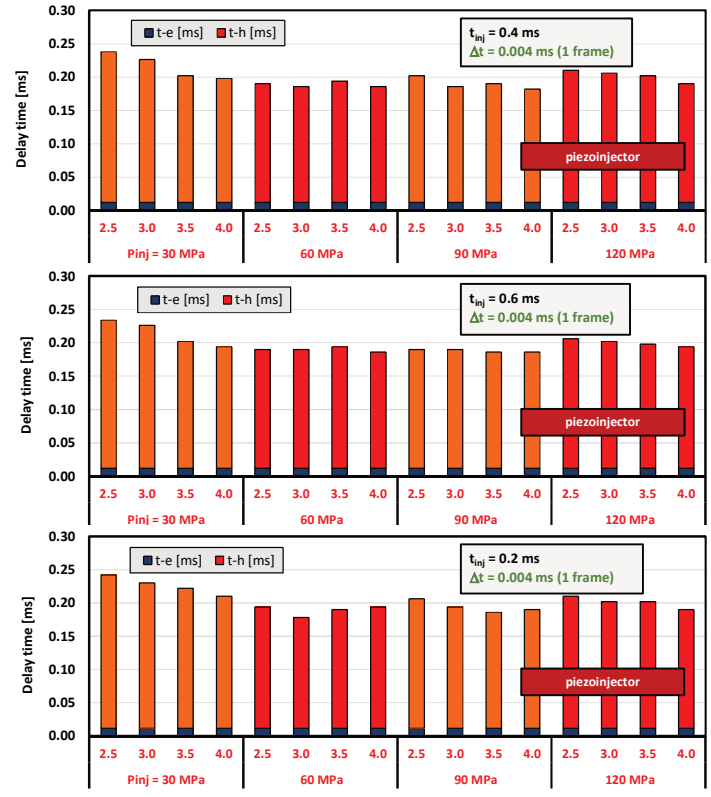

Fig. 6. Analysis of the times of injection delay at variable pressure of fuel injection and backpressure of air during piezoelectric injector operation
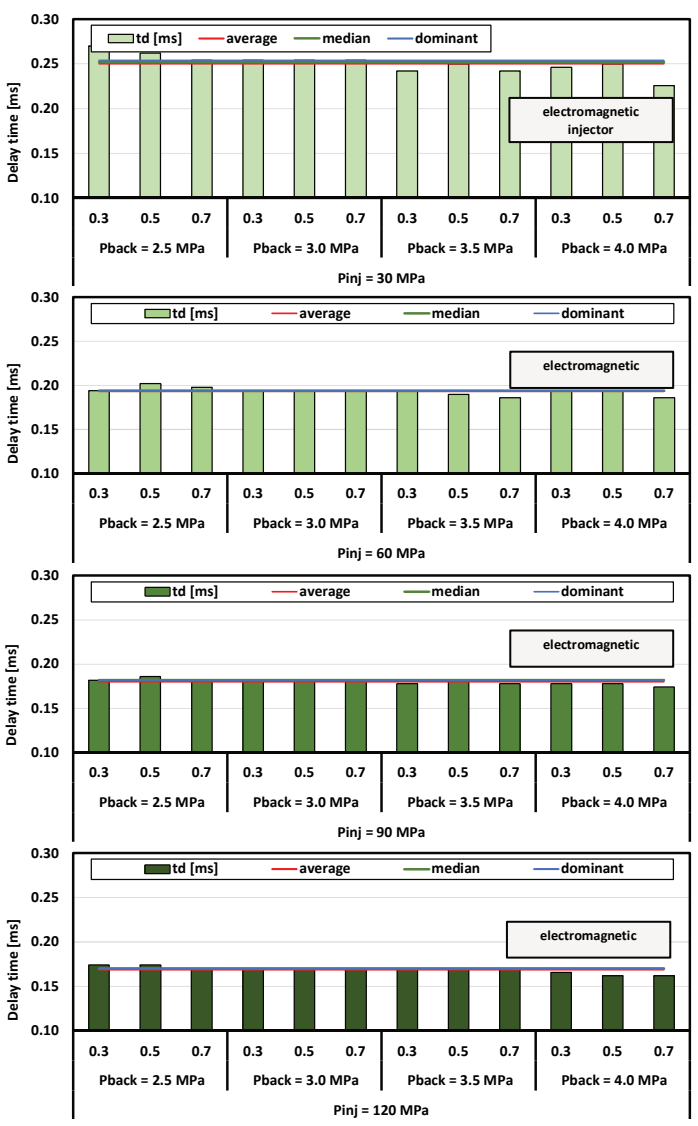

Fig. 7. Comparison of the times of fuel injection delays for electromagnetic injector for constant fuel injection pressure at variable backpressure and different energizing time

For a piezoelectric injector there is also no clear trend indicating the impact of input signal time on the injector response. This type of data presentation (Figs 7,8 ) indicates the trends mentioned earlier in the positive influence of backpressure on the value of injection delay. 

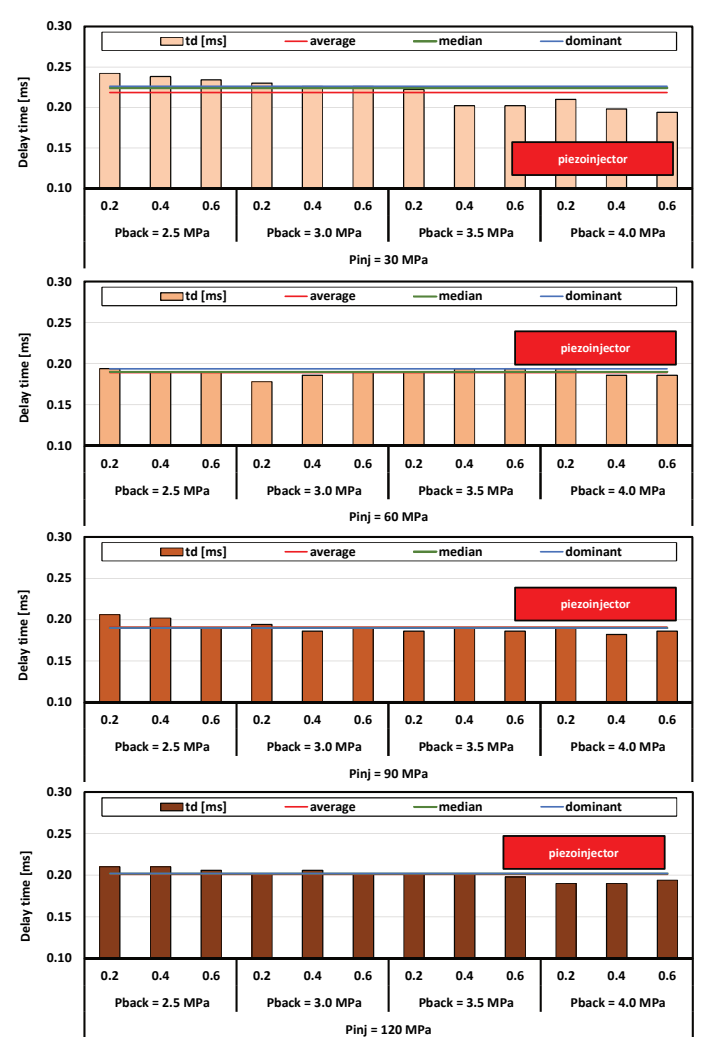

Fig. 8. Comparison of the times of fuel injection delays for piezoinjector for the constant fuel injection pressure at variable backpressure and different energizing times

\section{An attempt to describe in mathematical terms the fuel flow delay}

\subsection{The impact of the fuel pressure on the delay of fuel outflow}

The next stage of the study was an attempt at a mathematical description enabling obtaining the estimated value of the injection delay without the necessity to conduct tests. For this purpose the results were summarized and an attempt was made to assign the function best reflecting a trend that occurs between the fuel outflow delay and injection control parameters.

The first selected value, in relation to which the analysis was performed, was the injection pressure (for constant energizing time and backpressure) shown in Figs 9 and 10, for electromagnetic and piezoelectric injector, respectively. This analysis was presented for the mean, dominant and median value of injection delay time obtained from investigations. As the describing dependencies of this relation were selected linear, power and polynomial functions. For so selected functions the coefficient of determination was determined and, on this basis, the correctness of the description of this phenomenon with the use of indicated functions was estimated.

In all cases of results achieved for an electromagnetic injector, the power and polynomial functions produce a similar value of the coefficient of determination. The highest value was obtained for mean values described by the polynomial function of degree 2 and it amounted to $\mathrm{R}^{2}=$ 0.977. Matching at this level is satisfactory for polynomial and exponential functions.

The same analysis procedure was performed for piezoinjectors. In this case, the descriptions of the linear and power functions were characterized by a coefficient of determination the values of which pointed out to insufficient the phenomenon description using these functions. Again, as in the case of electromagnetic injector, the best match was obtained using a polynomial function, however, the best match was obtained on the basis of the dominant, where $\mathrm{R}^{2}=1$. For piezoelectric injectors, the analysis using various statistical measures indicates different characteristics of the dependency, which confirms the previous conclusion indicating the randomness of the injector operation.

The data analysis indicated the trend of changes for the electromagnetic injector was shown in Fig. 11. For the lowest injection pressure, the greatest differences were found for points with extreme backpressure values in the test chamber. This area alone has a clear tendency to show dependence on the backpressure.

It was assumed that the fuel injection time does not affect the opening delay of the electromagnetic injector. In this aspect, the opening delay of the injector $t_{d}$ was related to the fuel pressure $\mathrm{P}_{\mathrm{inj}}$ and the back pressure of the medium $\mathrm{P}_{\mathrm{b}}$ (Fig. 11).

Due to the specificity of injection delay time, analysis was divided into two stages:

1) for injection pressure of $30 \mathrm{MPa}$ - within this scope only the dependence on the back pressure of the medium was shown;

2) for injection pressure above $30 \mathrm{MPa}$ : within this scope the mathematical relationship between both, the fuel pressure and the pressure of the medium was determined. The results of these tests are presented in the following sections. a)

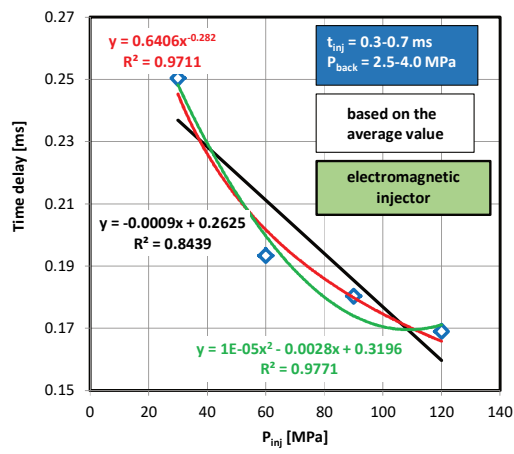

b)

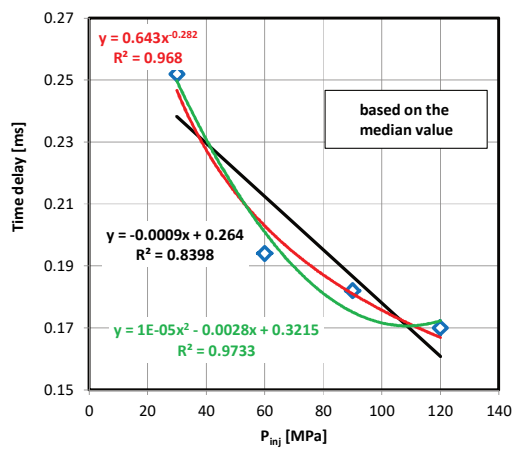

c)

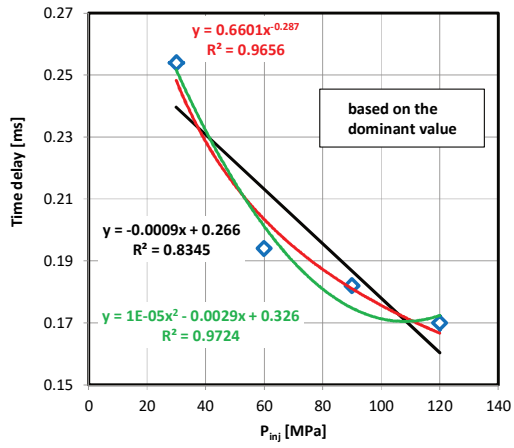

Fig. 9. Determination of mathematical models for electromagnetic injectors flow delay based on: a) mean values, b) values of the median, c) values of dominant, assuming independence of delay time from the time of the injection and backpressure 
a)

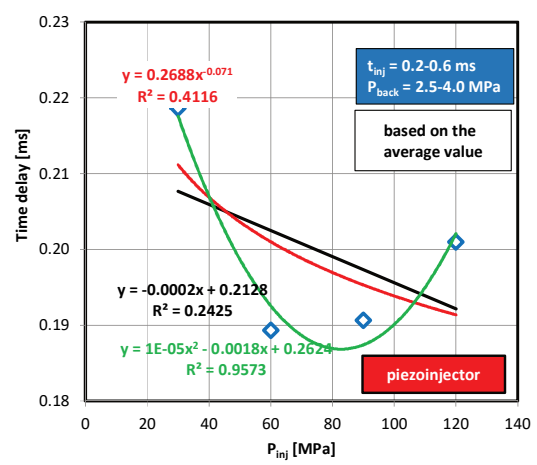

b)

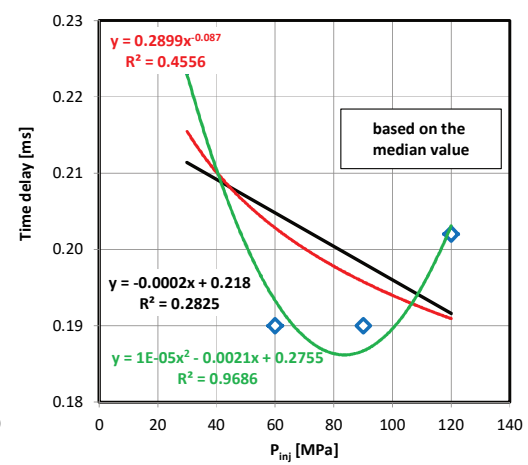

c)

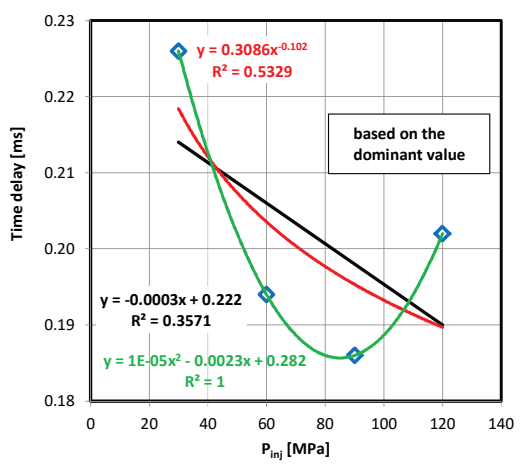

Fig. 10. Determination of mathematical models for piezoelectric injectors flow delay based on: a) mean values, b) values of the median, c) values of dominant, assuming independence of delay time from the time of the injection and backpressure

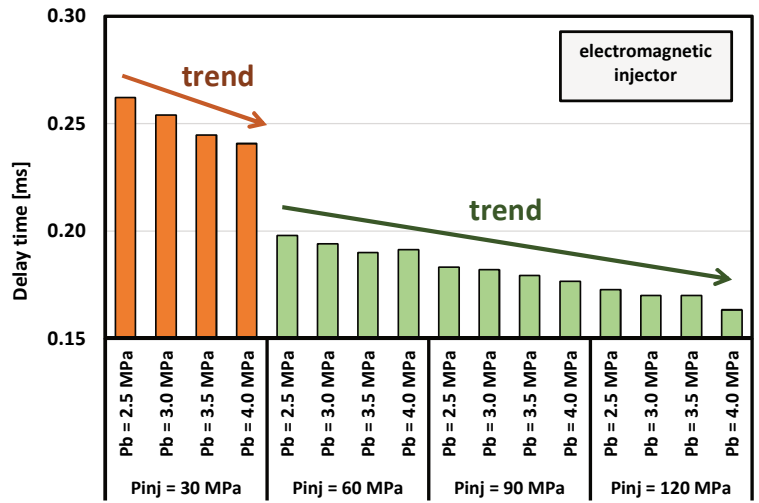

Fig. 11. Dependency of the delay time of opening of the electromagnetic injector from the injection time and backpressure of the medium

\subsection{The impact of the backpressure of the medium on the delay of fuel outflow}

The earlier assumptions about the parameters in relation to which a mathematical dependency is to be constructed require an initial estimate of the average step of change. Figure 12 shows a summary that provides information about the average change of the injection delay in relation to backpressure. A linear relationship was used to describe the mean change in injector response and the coefficient of determination was checked, which for both electromagnetic and piezoelectric injectors obtained a value of 0.98 . The description of such a relationship was considered to be sufficient and further analysis was carried out to obtain an empirical equation describing the injection delay for the indicated area of operation of the injector.

\subsection{A mathematical formula of the of fuel outflow dependency from the injector}

Based on the previous analyzes performed within the study and the information that delay at low pressure values mainly depends on the value of backpressure of the medium, its mathematical form was determined.

For both types of injectors one type of equation was adopted, taking into account the value of backpressure of the medium (at a constant fuel pressure $\mathrm{P}_{\text {inj }}=30 \mathrm{MPa}$ ) supplemented with the following coefficients: slope of line ' $a$ ' and exponent ' $b$ '. The general equation is proposed arbitrary in the following form:

$$
\mathrm{t}_{\mathrm{d}(\text { Pinj }=30 \mathrm{MPa})}=\mathrm{a} \cdot \mathrm{P}_{\mathrm{b}}^{\mathrm{b}}
$$

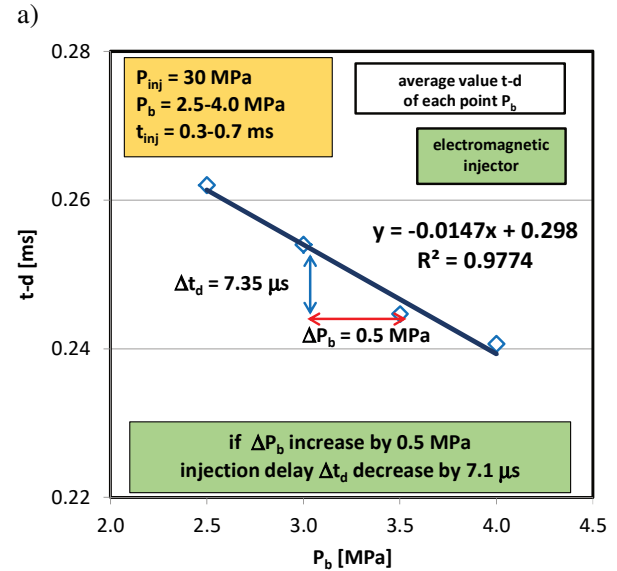

b)

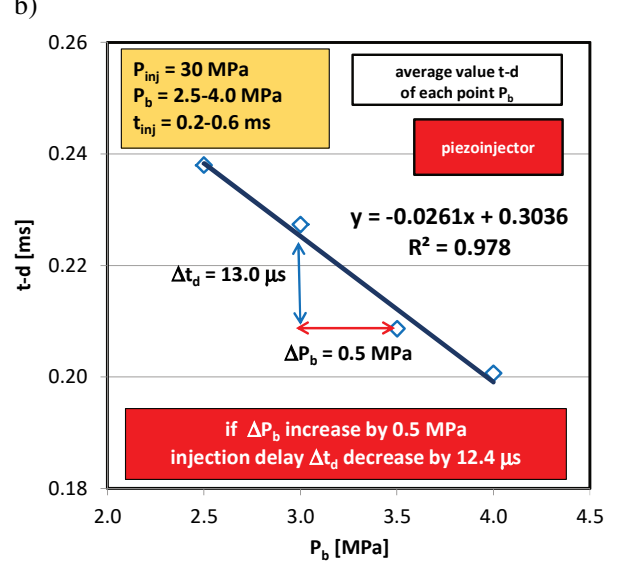

Fig. 12. Analysis of the change of fuel injection delay during changing the backpressure of the medium $\left(\mathrm{P}_{\text {inj }}=30 \mathrm{MPa}\right)$ on the basis of the average values of fuel injection using injectors: a) solenoid, b) piezoelectric

Determination of ' $a$ ' and ' $b$ ' coefficients requires the use of pseudo-optimization methods (which are here accepted as searching for proper function form and determination as the function's parameters). The optimization criteria are presented in the form of a decision to be minimized $f(U)$, expressed as the sum of squares of differences of the individual values of delays of fuel flow dependent only on the backpressure of air, designated experimentally and based on equation (3).

A mathematical model contains the decision variables, constraints, and decision-making function:

- decision variables: $a, b$ - coefficients of the equation (3), 
- constraint (designating the range of acceptable solutions): $a, b \neq 0$, values can be negative and positive, excluding zero (such value eliminates the equation),

- decision-making function: $\mathrm{U}(\mathrm{a}, \mathrm{b})$ fulfils the constraint, for which the decision-making function $f(U)$ reaches a minimum:

$$
\mathrm{f}(\mathrm{U})=\sum_{\mathrm{i}=1}^{\mathrm{n}}\left(\left(\mathrm{t}_{\mathrm{d}}\right)_{\mathrm{ci}}-\left(\mathrm{t}_{\mathrm{d}}\right)_{\mathrm{i}}\right)^{2} \rightarrow \min
$$

where $\left(t_{d}\right)$ - is the calculated delay value for given data of the parameters $\mathrm{P}_{b},\left(\mathrm{t}_{\mathrm{d}}\right)_{\mathrm{i}}$ - means a test value of the delay for the same values of $P_{b} ; i-$ is a subsequent value.

Using a solver implemented in MS Excel, a nonlinear solution to the task with nonlinear constraints was obtained. As mentioned earlier for this case only data used for injection pressure $30 \mathrm{MPa}$ were used.

This procedure is presented in Fig. 13, where the dependence (3) is considered as the optimization criterion of the equation. The coefficient of determination value for the piezo-injector was found to be satisfactory for the result obtained. In case of the electromagnetic injector, the result is unsatisfactory even to estimate the injection delay. After obtaining the results and their qualitative assessment, it was decided to move on to the next stage in which the empirical equation was extended by the injection pressure parameter.
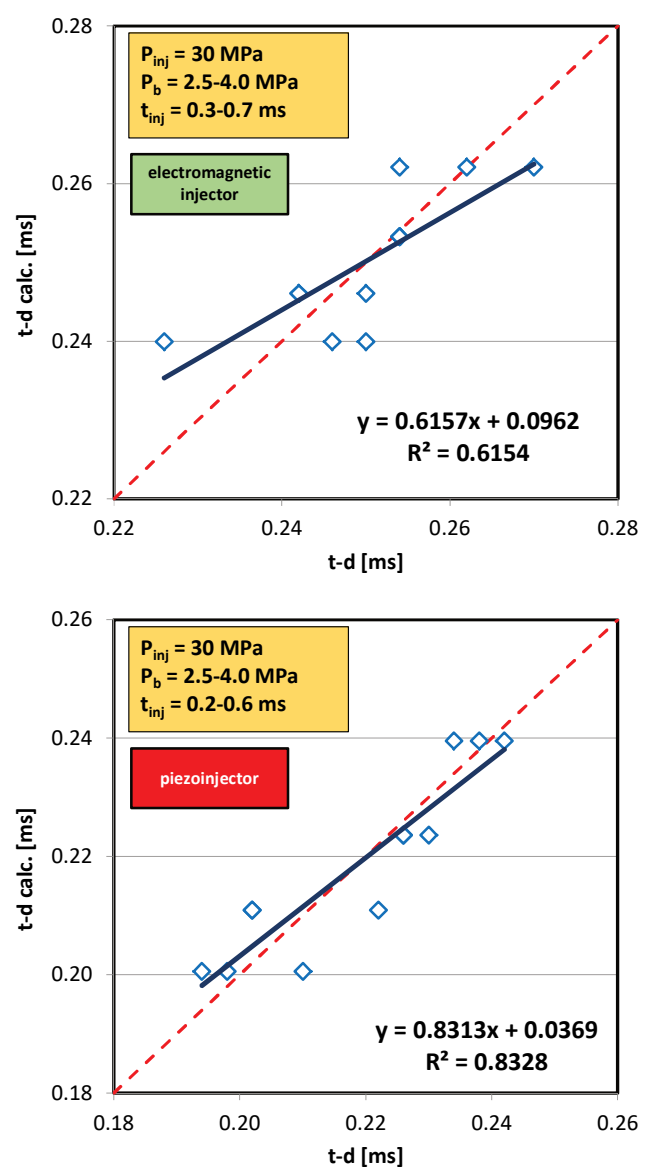

Fig. 13. The dependency of the fuel injection delay determined experimentally and analytically (conditions of the analysis are given in the figure; equation for electromagnetic injector: $\mathrm{t}_{\mathrm{d}}=0.3112 \cdot \mathrm{P}_{\mathrm{b}}{ }^{-0.187}$; equation for piezoinjector: $\mathrm{t}_{\mathrm{d}}=0.3387 \cdot \mathrm{P}_{\mathrm{b}}^{-0.378}$ )
Analysis of fuel injection delay at higher pressure values (Fig. 14) of fuel makes it possible to make the delay time dependent on two parameters: fuel pressure and backpressure of the medium. Using function:

$$
\mathrm{t}_{\mathrm{d}(\text { Pinj }>30 \mathrm{MPa})}=\mathrm{a} \cdot \mathrm{P}_{\mathrm{b}}^{\mathrm{b}} \cdot \mathrm{P}_{\mathrm{inj}}{ }^{\mathrm{c}}
$$

and the optimization criterion given in equation (4), the coefficients "a" "b" and "c" were determined making it possible to determine the correlation between experimental and calculated delay values. Coefficient of determination is $\mathrm{R}^{2}=0.94$, indicating a good match of the obtained coefficients with the experimental fuel injection delay.

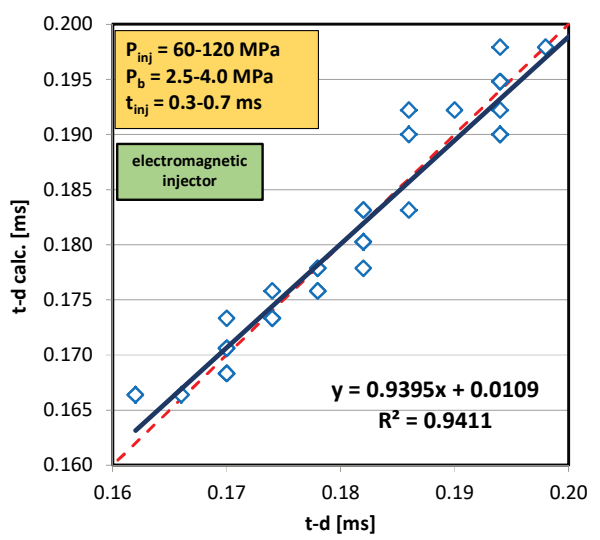

Fig. 14. Dependency of experimentally and analytically determined delay of fuel injection from electromagnetic injectors from fuel pressure and backpressure of the medium (conditions of analysis are given in the figure)

Table 5. The parameters characterizing fuel injection delay for diesel engines: piezo- and solenoid injectors

\begin{tabular}{|l|c|c|}
\hline & $\begin{array}{c}\text { Electromagnetic } \\
\text { injector }\end{array}$ & Piezoinjector \\
\hline Maximum injection delay & $0.27 \mathrm{~ms}$ & $0.24 \mathrm{~ms}$ \\
\hline Delay ratio EM/P $(\mathrm{P} / \mathrm{EM})$ & $1.125(1)$ & $1(0.888)$ \\
\hline $\begin{array}{l}\text { Increase } \Delta \mathrm{t}_{\mathrm{d}} \text { if } \Delta \mathrm{P}_{\mathrm{b}} \text { increase } \\
\text { by 0.5 MPa }\end{array}$ & $-7.35 \mu \mathrm{s}$ & $-13.0 \mu \mathrm{s}$ \\
\hline $\begin{array}{l}\text { Equation of injection delay } \\
\left.\text { (only for } \mathrm{P}_{\text {inj }}=30 \mathrm{MPa}\right)\end{array}$ & $\mathrm{t}_{\mathrm{d}}=0.3112 \cdot \mathrm{P}_{\mathrm{b}}^{-0.187}$ & $\mathrm{t}_{\mathrm{d}}=0.3387 \cdot \mathrm{P}_{\mathrm{b}}{ }^{-0.378}$ \\
\hline $\begin{array}{l}\text { Coefficient } \\
\text { of determination, } \mathrm{R}^{2}\end{array}$ & 0.6154 & 0.8328 \\
\hline $\begin{array}{l}\text { Equation of injection delay } \\
\left.\text { (for } \mathrm{P}_{\text {inj }}>30 \mathrm{MPa}\right)\end{array}$ & $\mathrm{t}_{\mathrm{d}}=0.469 \cdot \mathrm{P}_{\mathrm{b}}{ }^{-0.0869}$ & no correlation \\
\hline $\begin{array}{l}\text { Coefficient } \\
\text { of determination, } \mathrm{R}^{2}\end{array}$ & 0.9411 & - \\
\hline
\end{tabular}

Full analysis of the parameters of fuel injection delay and the process of optimization are presented in Table 5. A higher coefficient of determination was obtained when mathematical dependence was created for piezo-injectors, with the dependency of injection delay time only from backpressure (at injection pressure of $30 \mathrm{MPa}$ ).

The dependency describing the injection delay from the fuel pressure and the backpressure of the medium was determined only for electromagnetic injectors for the fuel pressure range above $30 \mathrm{MPa}$.

\section{Summary}

An electric and optical analysis of the gasoline injection delay makes it possible to determine the parameters characterizing the process. The use of averaged data indicates that any increase in air pressure $\Delta \mathrm{P}_{\mathrm{b}}$ by $0.5 \mathrm{MPa}$ results in an 
increase in the operating delay of the electromagnetic injector by $5.65 \mu \mathrm{s}$. Using experimental research, the mathematical dependence of the delay in injector operation time from the air backpressure was determined, as shown in Table 5. These equations were obtained for relatively high values of coefficients of determination, which means a large convergence with the results of experimental research.

As the results of experimental studies different times of fuel injection delay relating to electromagnetic and piezoelectric injectors were obtained.

Experimental studies allowed some observations and conclusions to be made regarding diesel fuel injectors:

1. A delay in opening the injectors can be described with the use of mathematical models in which the fuel pressure and backpressure of the medium are taken into account. The operation analysis of the solenoid injectors supplying diesel fuel indicates the reduction of the fuel flow delay time from the injector with the increasing fuel pressure (within the range from 30 to $120 \mathrm{MPa}$ ). Delay of diesel fuel flow from piezoelectric injectors is reduced only in the range of
30-60 MPa. For further increasing fuel pressure, the delay is constant or slightly increased.

2. At a pressure of $30 \mathrm{MPa}$ the delay time for solenoid injectors of diesel fuel is proportional to the backpressure of air. Any increase in the backpressure of the air $\Delta \mathrm{P}_{\mathrm{b}}$ by $0.5 \mathrm{MPa}$ decreases the time of fuel flow delay by $7.35 \mu \mathrm{s}$.

3. At low values of fuel pressure $(30 \mathrm{MPa})$ the delay time for piezoelectric injectors of diesel fuel is proportional to the backpressure of air. Any increase in the backpressure of the air $\Delta \mathrm{P}_{\mathrm{b}}$ by $0.5 \mathrm{MPa}$ decreases the time of fuel flow delay by $13.0 \mu \mathrm{s}$.

4. Delay of fuel flow from diesel fuel injectors at fuel supply pressure above $30 \mathrm{MPa}$ is described by the dependence using $\mathrm{P}_{\text {inj }}$ and $\mathrm{P}_{\mathrm{b}}$ and as a result the high compatibility of the equation with the experimental results was obtained. Unfortunately, this dependence could only be determined for electromagnetic injectors.

\section{Nomenclature}

$\begin{array}{ll}\text { CI } & \text { compression ignition } \\ \text { CNG } & \text { compressed natural gas } \\ \text { CVC } & \text { constant volume chamber } \\ \text { DI } & \text { direct injection } \\ \text { ET } & \text { energizing time }\left(=\mathrm{t}_{\text {inj }}\right) \\ \text { LPG } & \text { liquefied petroleum gas }\end{array}$

CI compression ignition

CVC constant volume chamber

ET energizing time $\left(=\mathrm{t}_{\text {inj }}\right)$

LPG liquefied petroleum gas

$\begin{array}{ll}\mathrm{P}_{b} & \text { back pressure } \\ \mathrm{P}_{\text {inj }} & \text { injection pressure } \\ \mathrm{SI} & \text { spark ignition } \\ \mathrm{t}_{\mathrm{d}} & \text { total time of delay } \\ \mathrm{t}_{\mathrm{e}} & \text { electrical time delay } \\ \mathrm{t}_{\mathrm{h}} & \text { hydraulic delay time }\end{array}$

\section{Bibliography}

[1] DUAN, L., YUAN, S., HU, L. et al. Injection performance and cavitation analysis of an advanced $250 \mathrm{MPa}$ common rail diesel injector. International Journal of Heat and Mass Transfer. 2016, 93, 388-397.

[2] MAGNO, A., MANCARUSO, E., VAGLIECO, B.M. Experimental investigation in an optically accessible diesel engine of a fouled piezoelectric injector. Energy. 2014, 64, 842-852.

[3] SALVADOR, F.J., GIMENO, J., CARRERES, M., CRIALESI-ESPOSITO, M. Fuel temperature influence on the performance of a last generation common-rail diesel ballistic injector. Part I: Experimental mass flow rate measurements and discussion. Energy Conversion and Management. 2016, 114, 364-375.

[4] SKOWRON, M., PIELECHA, I. Analysis of injectors reaction on the external signals in direct injection systems. Jour-
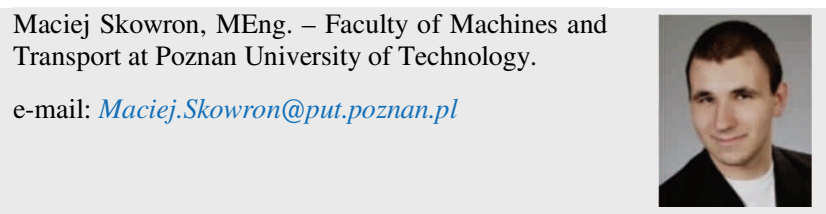

Prof. Krzysztof Wisłocki, DSc., DEng. - Faculty of Machines and Transport at Poznan University of Technology.

e-mail: Krzysztof.Wistocki@put.poznan.pl nal of Mechanical and Transport Engineering. 2016, 68(3), 39-51.

[5] SKOWRON, M., PIELECHA, I. Evaluation of difference between controling signal and injector response time in liquid fuel direct injection systems. Combustion Engines. 2015, 162(3), 327-334.

[6] SKOWRON, M., PIELECHA, I., WISŁOCKI, K. Transient states analysis of CI engine injectors with the use of optical methods. Publishing IOP Conf. Series: Materials Science and Engineering. 2016, 148, 1-8.

[7] YU, W., YANG, W., TAY, K. et al. Macroscopic spray characteristics of kerosene and diesel based on two different piezoelectric and solenoid injectors. Experimental Thermal and Fluid Science. 2016, 76, 12-23.

[8] ZHOU, L.Y., DONG, S.F., CUI, H.F. et al. Measurements and analyses on the transient discharge coefficient of each nozzle hole of multi-hole diesel injector. Sensors and Actuators A: Physical. 2016, 244, 198-205.

Ireneusz Pielecha, DSc., DEng. - Faculty of Machines and Transport at Poznan University of Technology.

e-mail: Ireneusz.Pielecha@put.poznan.pl

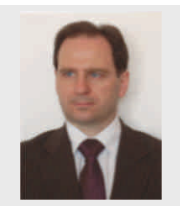

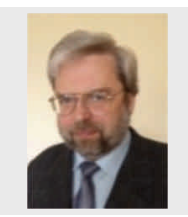

